Психология. Журнал Высшей школы экономики,

2011. T. 8, № 1. С. $89-100$.

\title{
ЭЛЕКТРОГРАФИЧЕСКИЕ КОРРЕЛЯТЫ РЕШЕНИЯ ЗАДАЧИ ЗРИТЕЛЬНОГО ПОИСКА У ЧЕЛОВЕКА
}

\author{
Н.С. ЕРМАЧЕНКО, А.А. ЕРМАЧЕНКО, А.В. ЛАТАНОВ
}

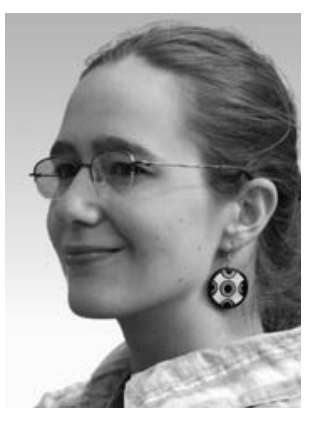

Ермаченко Наталья Сергеевна - аспирант биологического факультета МГУ им. М.В. Ломоносова. Область научных интересов - психофизиология, движения глаз, зрительное восприятие.

Контакты: natalia.msu@gmail.com

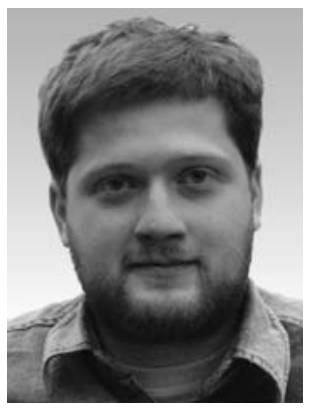

Ермаченко Александр Александрович - лаборант биологического факультета МГУ им. М.В. Ломоносова. Область научных интересов - информационные технологии, движения глаз, психофизиология.

Контакты: admin@neurobiology.ru

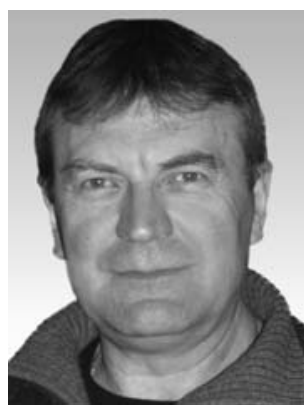

Латанов Александр Васильевич - профессор биологического факультета МГУ им. М.В. Ломоносова, доктор биологических наук, доцент. Область научных интересов - нейрофизиология, движения глаз, психофизиология, зрительное восприятие.

Контакты: latanov@neurobiology.ru 


\section{Резюме}

Авторы провели исследование динамики ЭЭГ в альфа-диапазоне при выполнении задачи зрительного поиска релевантного стимула (РC) среди многих нерелевантных. В качестве контроля использовали простое рассматривание нескольких одинаковых стимулов. Для верификации взора при поиске РС использовали метод видеоокулографии. Динамику ЭЭГ в альфа-диапазоне (десинхронизацию, Д) рассматривали как коррелят процессов внимания. Показана существенно бо́льшая, чем в контроле, Д после нахождения РС. Большая

выраженность Д при почске, по-видимому, связана с процессами оценки значимости стимула (при вовлечении произвольного модально-специфического внимания). При этом еще бо́льшая по амплитуде Д в лобных областях предположительно отражает значимость выполнения адекватной двигательной программы (при вовлечении префронтальных механизмов внимания).

Ключевъе слова: селективное внимание, зрительный поиск, десинхронизачия

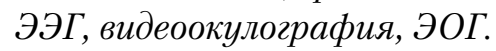

\section{Введение}

Зрительный поиск с участием движений глаз играет ключевую роль в повседневной деятельности человека. Из-за сложности реальной зрительной среды и ограниченности ресурсов зрительной системы при обработке зрительной информации решающим условием для успешности поиска является селективное выделение наиболее значимой информации. Такой выбор достигается смещением внимания в пределах зрительного поля, которое тесным образом связано с саккадическими движениями глаз (Findlay, 1997; Motter, Holsapple, 2007). Процесс распознавания зрительной информации происходит в период фиксаций взора, в течение которого зрительный объект проецируется на центральную ямку сетчатки. Таким образом, зрительно-моторная поисковая деятельность обеспечивается зрительным селективным вниманием, кото- рое включает разные уровни и компоненты в зависимости от специфики перцептивной деятельности.

M. Познер (Posner, 1980) предложил рассматривать внимание как систему контроля ментальных процессов, включающую три подсистемы: (1) ориентацию на сенсорное событие; (2) выделение зрительного сигнала для его распознавания и (3) поддержание активности коры головного мозга. Две первые подсистемы непосредственно связаны с движениями глаз: первая с процессами пространственного внимания, направляющего взор (путем генерации саккады) на интересующий объект; вторая обеспечивает детекцию зрительного сигнала в выделенной (в результате работы первой подсистемы) области зрительной сцены. Такая пространственно селективная детекция метафорично называется М. Познером «лучом прожектора» (метафора «прожектора», англ., spotlight metaphor), выделяющим из 
зрительной среды наиболее значимые участки.

В соответствии с современными представлениями (Wolfe, 1994; Motter, Holsapple, 2007; Hwang et al., 2009), зрительный поиск обеспечивается двумя факторами: первый зависит от стимулов, а второй от цели (задачи). Зрительные объекты, находящиеся в поле зрения, активируют восходящие процессы непроизвольного внимания (так называемое внимание «снизу-вверх», англ., bottom-up). Произвольное внимание определяется зрительной задачей (например, установление связи между целевым (искомым) объектом и локальными участками зрительной сцены) и представлено нисходящими процессами (так называемое внимание «сверху-вниз», англ. top-down). Анатомической базой этих процессов являются две морфофункциональные системы - задняя и передняя (Posner, Petersen, 1990). В коре мозга задняя система включает теменные, а передняя - префронтальные области. Эти системы взаимодействуют между собой через прямые (внутрикорковые) и опосредованные (с включением некоторых подкорковых образований) связи.

Идея Д. Линдсли (Lindsley, 1960) о том, что внимание отражается в сдвиге функционального состояния и, соответственно, в динамике ЭЭГ, в дальнейшем нашла многочисленные экспериментальные подтверждения. В большом числе работ, начиная с пионерских исследований Г. Уолтера (Walter, 1969), показана специфическая десинхронизация (уменьшение амплитуды) ЭЭГ в альфа-диапазоне, сопровождающая произвольную деятельность с вовлече- нием внимания (Shaw, 2003). Ментальные процессы, происходящие с вовлечением внимания (восприятие, распознавание, решение мнемонических и семантических задач, принятие решения), а также исполнительные компоненты целенаправленной деятельности (инициация, осуществление и подавление двигательных реакций) также вносят свой вклад в динамику ЭЭГ (Klimesch et al., 2007; Pfurtscheller, Klimesch, 1992; Pfurtscheller, Lopes da Silva, 1999). Таким образом, ЭЭГ-корреляты процессов внимания интерферируют с аналогичными коррелятами других компонентов произвольной деятельности.

При изучении процессов селективного зрительного внимания в парадигме произвольного зрительного поиска с использованием метода регистрации движений глаз обычно не регистрируют ЭЭГ. Это связано с тем, что движения глаз производят значительные артефакты, которые затрудняют выделение и анализ ЭЭГ-коррелятов произвольной деятельности. В такой парадигме о ментальной деятельности (в том числе и о внимании) можно судить лишь косвенно, основываясь на пространственно-временной динамике траектории взора. При осуществлении какой-либо произвольной деятельности (перцептивной, мнемонической или семантической) нельзя исключить ситуации, когда фокусы взора и внимания, обеспечивающего эту деятельность, не совпадают. В таких случаях информация о положении взора не позволяет судить о ментальной активности, связанной с событиями, происходящими в пределах фокуса взора. 
С другой стороны, использование относительно простых парадигм (без поиска и, соответственно, без движений глаз), в которых ограничиваются регистрацией только электрофизиологических показателей (ЭЭГ, вызванных потенциалов, потенциалов, связанных с событиями), существенно сужает круг задач для исследования произвольной деятельности. Очевидным преимуществом такого подхода является исключение исполнительных глазодвигательных реакций, производящих существенные артефакты и добавляющих дополнительные компоненты к электрографическим эффектам, связанным с ментальной деятельностью.

В силу различных причин в большинстве работ по исследованию зрительного селективного внимания использовали парадигмы, моделирующие относительно простые компоненты реальной сложной зрительной среды. Такая редукция, несомненно, исключает неопределенность при интерпретации регистрируемых эффектов, однако не позволяет исследовать произвольную деятельность в условиях, приближенных к реальным. Например, стандартная методика для исследования компонентов вызванных потенциалов (в частности, Р300) не предполагает одновременного предъявления нескольких стимулов. С другой стороны, исследования в рамках парадигмы зрительного поиска, как правило, ограничиваются лишь регистрацией движений глаз и анализом их параметров (Duncan, Humphreys, 1989; Motter, Holsapple, 2007). Редкое исключение составляют работы (Gomarus et al., 2006), в которых испытуемым предъявляли несколько (до четырех) стимулов одновременно: в этом случае для решения задачи зрительного поиска испытуемый должен был совершить не более одной саккады. В таких условиях оказалось возможным усреднять потенциалы, связанные с событиями, и десинхронизацию ЭЭГ, связанную с событием, в определенном частотном диапазоне относительно момента позиционирования взора на каком-либо стимуле, т.е. непосредственно после завершения саккады в его направлении.

Целью нашей работы является интеграция стандартных методов видеоокулографии и электроэнцефалографии для синхронной регистрации траектории взора и электрографических показателей (ЭЭГ, ЭОГ). Такая интегрированная методика использовалась нами для исследования динамики ЭЭГ в альфа-диапазоне при выполнении задачи произвольного зрительного поиска в условиях, приближенных к естественным.

\section{Методика}

В экспериментах участвовали 16 испытуемых (9 мужчин и 7 женщин) в возрасте 21-25 лет. Все испытуемые имели нормальное зрение и не страдали неврологическими заболеваниями. Эксперименты проводились с соблюдением основных правил биоэтики.

Использовали экспериментальную парадигму зрительного поиска, в которой испытуемых инструктировали находить релевантный стимул (РС), предъявляемый одновременно с 45-57 нерелевантными стимулами (нРС) (рисунок 1). 
Стимулы, используемые в экспериментах: слева - РС, справа - нРС

Рисунок 1
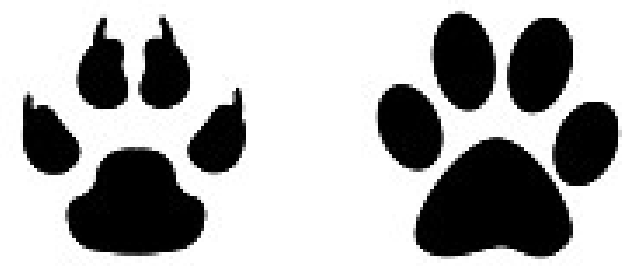

Во время выполнения задачи регистрировали ЭЭГ, электроокулограмму (ЭОГ), а также движения левого глаза методом видеоокулографии. Стимулы предъявляли на мониторе Samsung SyncMaster 940N, располагаемом в 45 см от глаз испытуемого. Биопотенциалы регистрировали монополярно при помощи электроэнцефалографа Medicor 16S (Венгрия) от 8 электродов $(\mathrm{Ag} / \mathrm{AgCl})$, расположенных по системе «10-20\%» (стандартные отведения F3, F4, C3, C4, P3, P4, O1, O2), относительно объединенного ушного электрода $(\mathrm{Ag} / \mathrm{AgCl})$. Фильтрацию биопотенциалов осуществляли в диапазоне 0.5-30 Гц, также применяли режекторный фильтр для частоты 50 Гц. ЭОГ регистрировали биполярно двумя чашечковыми электродами $(\mathrm{Ag} / \mathrm{AgCl})$, расположенными на внешней и внутренней орбитах левого глаза (горизонтальная составляющая ЭОГ), и двумя такими же электродами, расположенными над и под орбитой левого глаза (вертикальная составляющая ЭОГ). Полоса пропускания потенциалов ЭОГ составляла 0.16-15 Гц. Биопотенциалы оцифровывали аналого-цифровым преобразователем L-154 (фирма LCard, Россия) с частотой 512 Гц под управлением программной среды CONAN (Кулаичев,
2002). Видеоизображение глаза регистрировали камерой АCE-560B-92 (CHPI) с частотой 25 Гц. Видеорегистрация производилась в условиях инфракрасной (ИК) подсветки глаза ИК-светодиодами (TSAL5100, $\lambda_{\text {peak }}=$ $=940$ нм). Камеру помещали в тефлоновый корпус и закрепляли на специальном шлеме, одеваемом на голову испытуемого. Внутри корпуса устанавливали специальные светодиоды (TSAL5100, $\lambda_{\text {peak }}=940$ нм) для ИК-засветки камеры на 100 мс с целью синхронизации видеосигнала с ЭЭГ и ЭОГ. Камеру подключали к компьютеру через USB-порт, и получаемые с нее кадры в режиме реального времени записывали на жесткий диск в программной среде MS Video Maker. Перед объективом камеры устанавливали тепловой фильтр, пропускающий свет только в ИК-диапазоне (более 940 нм), что позволило значительно увеличить контраст между зрачком (самая темная область) и остальной частью изображения глаза. Все потоки данных (ЭЭГ, ЭОГ, видеорегистрация, предъявление изображений) синхронизировали оригинальными аппаратными средствами.

В экспериментах испытуемым предъявляли по 100 чередующихся контрольных (К) и тестовых (Т) 
Пример тестового изображения с набором стимулов; стрелкой отмечен РС. Линиями

Рисунок 2 обозначены саккады, а кружками - фиксации взора, регистрируемые при поиске РС

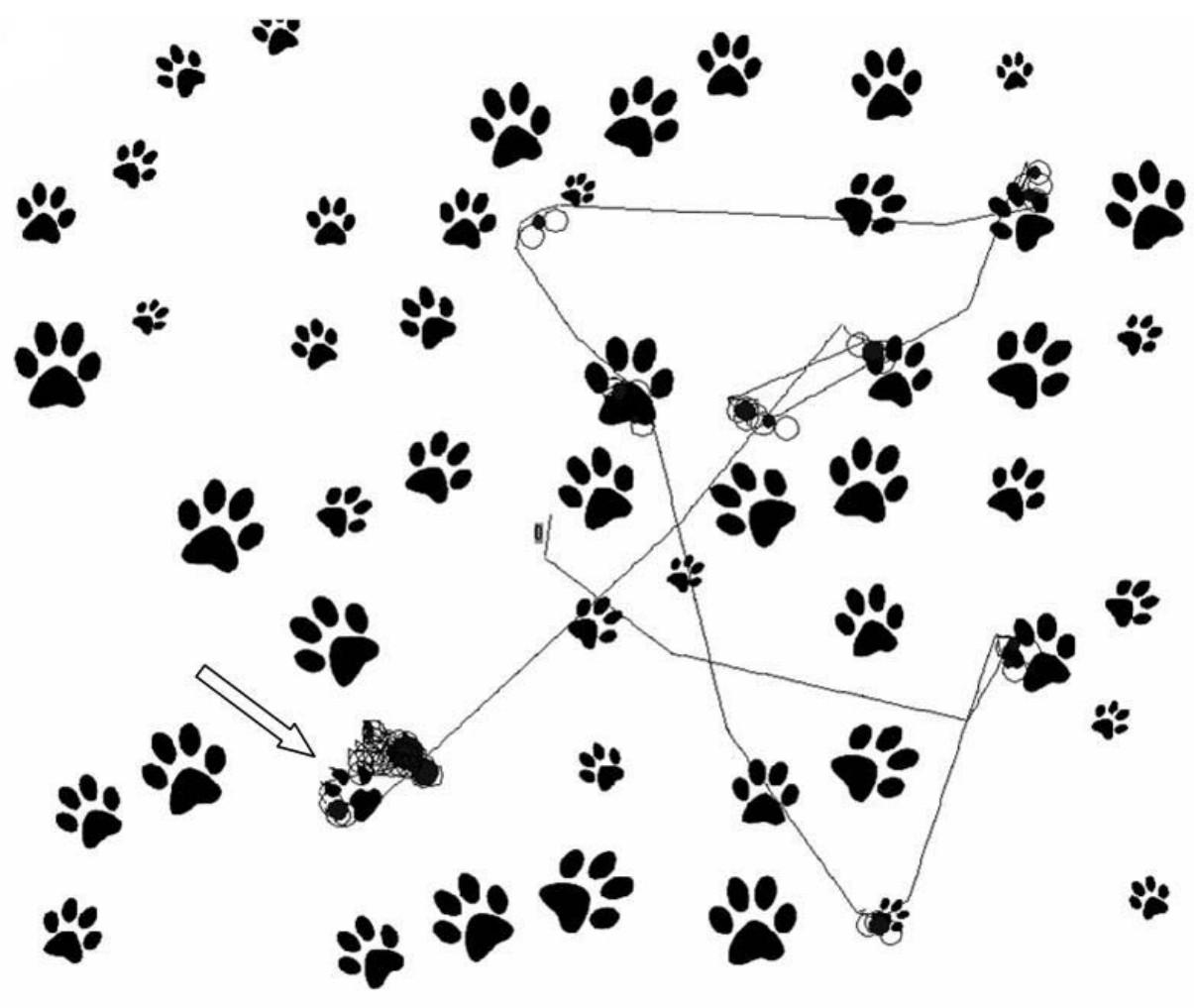

изображений (рисунок 2). На К предъявляли четыре стимула размером 2 угл. град. (по форме эквивалентные PC на Т), расположенные в углах воображаемого прямоугольника со сторонами $8 \times 6$ град. по центру зрительного поля. На Т размер (в пределах $1-3^{\circ}$ ) и ориентацию (повороты в пределах $\pm 60^{\circ}$ относительно вертикали) всех нРС и одного PC меняли псевдослучайным образом. Положение РС среди нРС, а также число последних меняли в псевдослучайном порядке. Стимулы могли касаться друг друга, но не перекрываться.
При экспозиции К испытуемых инструктировали последовательно фиксировать взор примерно на 2 с на каждом стимуле произвольным образом. После выполнения четырех фиксаций экспериментатор включал следующее Т. При экспозиции Т испытуемых инструктировали находить РС и фиксировать на нем взор примерно на 2 с. После этой фиксации экспериментатор включал следующее К. Треки движений взора при просматривании К использовали для калибровки координат взора, а динамику ЭЭГ при фиксации последнего стимула на К - 
в качестве контроля при сравнении с динамикой ЭЭГ в задаче зрительного поиска.

При выполнении задачи зрительного поиска анализировали динамику ЭЭГ в тот период, когда испытуемые находили единственный РС и фиксировали на нем взор. При этом до включения следующего К испытуемые не совершали ни одной саккады.

С использованием видеозаписи движений глаза приблизительно определяли момент окончания поиска РС. Точность такой синхронизации составляла 80 мс. Более точное определение момента окончания поиска осуществляли по последней саккаде (по пику ЭОГ), после которой испытуемый фиксировал взор на РС. Точность определения этого пика составляла 1/30 с (из-за искажения ЭОГ в результате фильтрации с использованием фильтра нижних частот 15 Гц).

При анализе ЭЭГ исключали фрагменты с артефактами различной природы с амплитудой более 100 мкВ. Динамику ЭЭГ в альфа-диапазоне (8-13 Гц) анализировали с использованием метода вычисления десинхронизации/синхронизации по алгоритму Г. Пфуртшеллера (Pfurtscheller, Aranibar, 1977). Число реализаций для усреднения динамики ЭЭГ при поиске составило 64-87 у разных испытуемых (в среднем 73) и 51-81 (в среднем - 65) в контроле. Анализ данных производили с помощью оригинальных программ, разработанных в программной среде Matlab (The Mathworks, Inc., Natic, MA) с использованием части функций программной системы EEGLAB (Delorme, Makeig, 2004).

\section{Результаты}

После того как испытуемые в результате поиска находили РС (фиксировали на нем взор), во всех отведениях отмечалось снижение амплитуды ЭЭГ в альфа-диапазоне (десинхронизация, Д) в течение примерно 2 с (рисунки 3, 4). Подобная динамика ЭЭГ выявлена у всех испытуемых. В контроле после последней фиксации также отмечалась Д, однако ее выраженность (продолжительность и амплитуда) была существенно меньше, чем при поиске РС (влияние фактора «парадигма» (поиск и контроль) $-\mathrm{F}=32.05, p<0.0001, \mathrm{df}=240$, рисунок 4). В контроле в период времени от -200 до 100 мс (относительно момента начала фиксации) регистрировали значительную синхронизацию (рисунок 4), которая соответствовала артефакту (ЭОГ) от саккад с амплитудой 6-8 град. Подобная синхронизация регистрировалась и при осуществлении поиска, однако ее амплитуда была существенно меньше из-за меньших амплитуд саккад при поиске. Как в контроле, так и при поиске амплитуда такой синхронизации снижалась в каудальном направлении.

Для сравнения Д в контроле и при поиске вычисляли интегральную величину степени Д как площадь под отрицательной частью кривой зависимости Д от времени в интервале 0-2000 мс (в период фиксации взора на стимулах, рисунок 5). Амплитуда и продолжительность Д при поиске были больше в лобных по сравнению с другими отведениями (влияние фактора «отведение» по интегральной величине Д $\mathrm{F}=2.287, p<0.032$, $\mathrm{df}=7.120$, рисунки 3,5$)$. Выраженность 


\section{Фрагмент записи биопотенциалов}

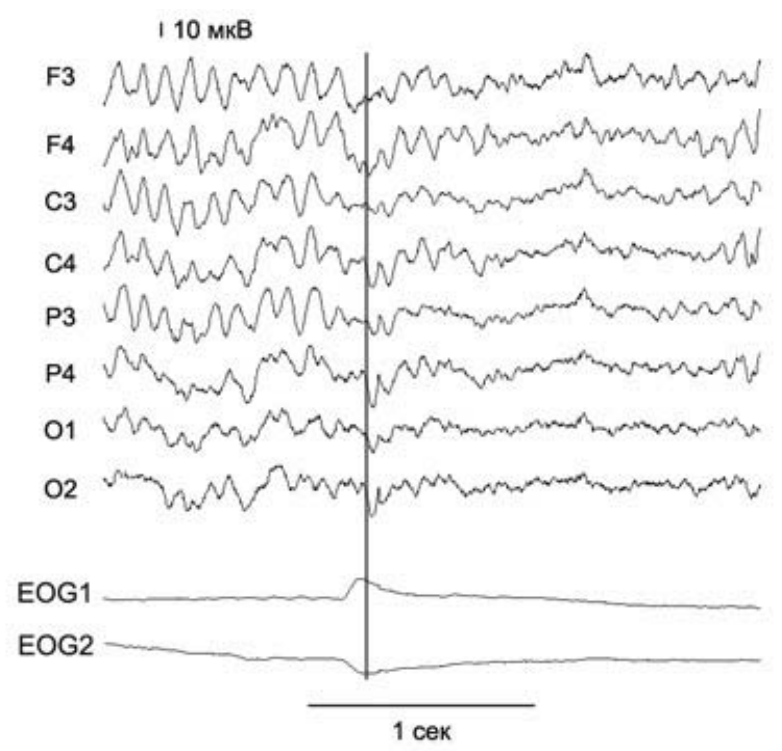

Примечание. Вертикальной линией обозначен момент нахождения РС (начало фиксации взора на нем), установленный по максимумам EOG1 (вертикальная компонента ЭОГ) и EOG2 (горизонтальная компонента ЭОГ). ЭЭГ отфильтрована в альфа-диапазоне.

Д в контроле мало изменялась по отведениям (влияние фактора «отведение» по интегральной величине Д $\mathrm{F}=0.115, p<0.996, \mathrm{df}=7.120$, рисунки 4, 6).

Во всех отведениях Д при поиске оказалась существенно выше, чем в контроле (рисунок 6). При этом наибольшие различия (в среднем более чем в 2 раза) отмечались в лобных отведениях, в остальных отведениях - в среднем в 1.5 раза.

\section{Обсуждение}

В данной работе мы продемонстрировали возможности метода, основанного на синхронизации отдельных потоков данных (видеорегистра- ция движений глаз и ЭЭГ). С одной стороны, метод позволяет предъявлять испытуемому сложные изображения, навигация взором по которым с целью поиска «нужных» стимулов требует совершения многих саккад. С другой стороны, при этом представляется возможным выделять фрагменты ЭЭГ, совпадающие во времени с фиксациями взора на воспринимаемых стимулах. Метод видеоокулографии (совместно с электроокулографией) используется для верификации положения взора испытуемого при выполнении различных задач, вовлекающих движения глаз. При этом динамика синхронно регистрируемой ЭЭГ объективно отражает функциональное 
Рисунок 4

Усредненная по всем испытуемым динамика Д в альфа-диапазоне, полученная по методу Г. Пфуртшеллера (Pfurtscheller, Aranibar, 1977)
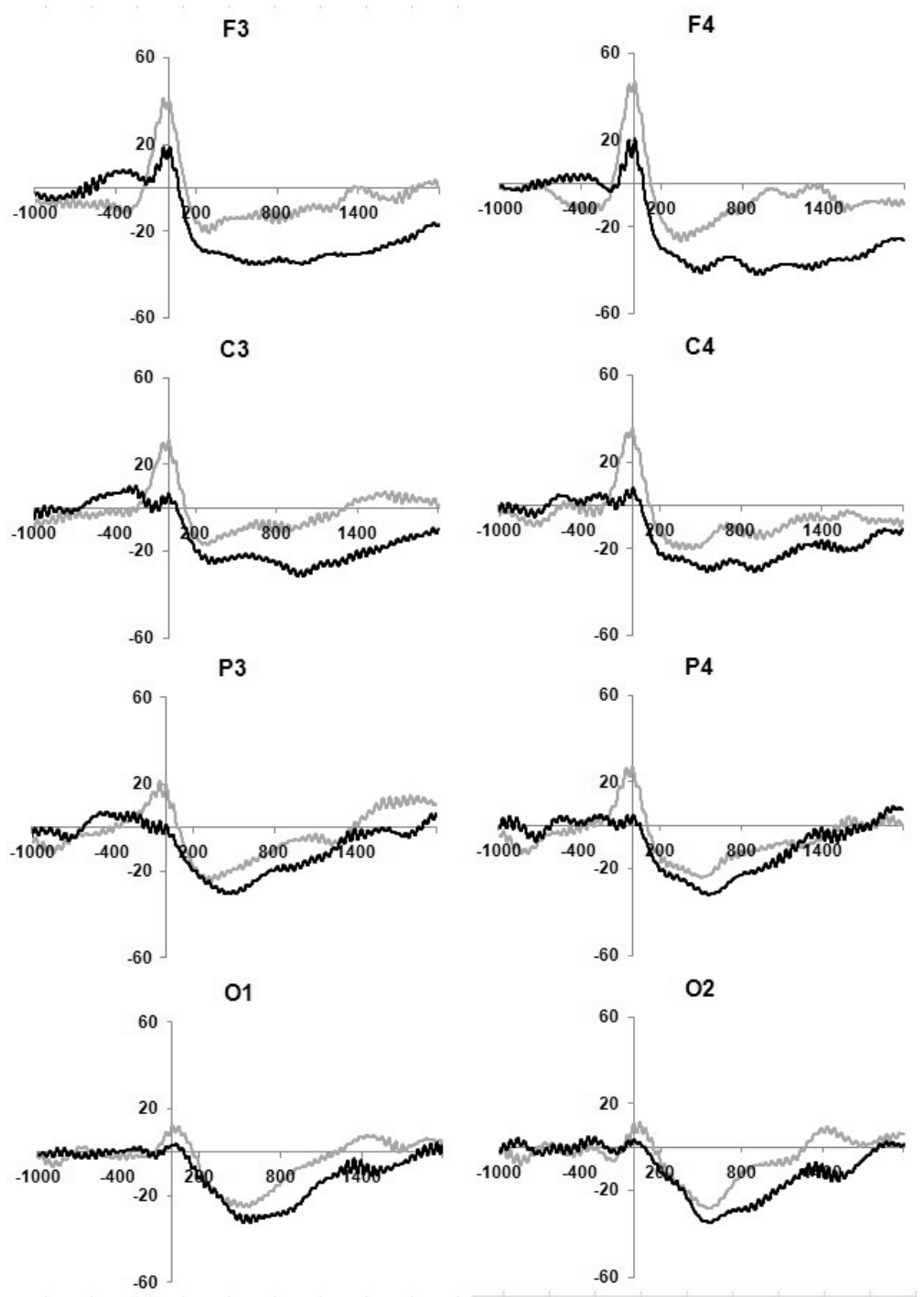

Примечание. По оси абсцисс - время в мс (0 - момент, соответствующий максимуму саккады, определяемому по ЭОГ, рисунок 3 ); по оси ординат - относительная амплитуда Д по сравнению с базовым уровнем (в интервале от -1000 до 0 мс), \%. Темная линия - Д при предъявлении Т, светлая линия - Д при предъявлении К. Положительные значения соответствуют синхронизации (увеличение амплитуды потенциалов в альфа-диапазоне), отрицательные - десинхронизации (уменьшение амплитуды потенциалов в альфа-диапазоне). 
Метод вычисления интегральной величины степени Д (отведение F3)

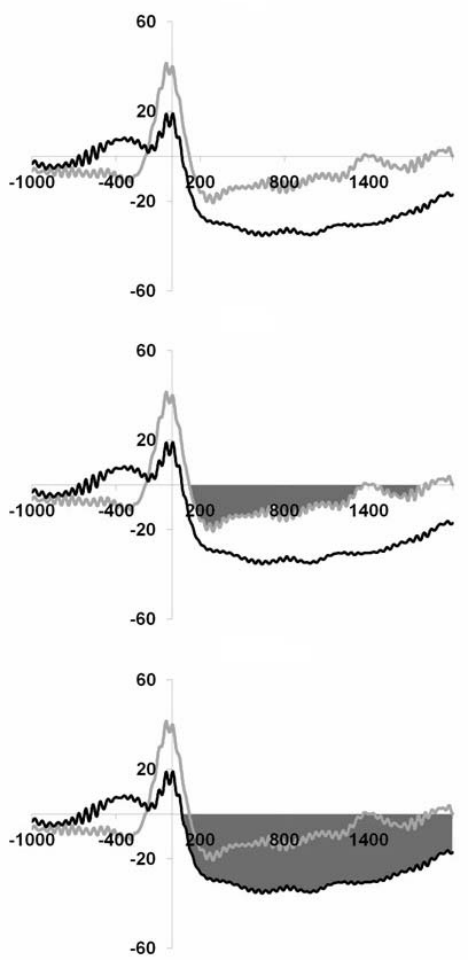

Примечание. Вверху - динамика Д в контроле и при поиске; в середине - площадь под кривой Д в контроле; внизу - площадь под кривой Д при поиске. Остальные обозначения как на рисунке 4.

состояние ЦНС испытуемых, сопровождающее их произвольную деятельность.

Согласно нашим результатам, нахождение РC приводит к выраженной Д в диапазоне альфа-ритма, что согласуется с недавними экспериментальными данными (Klimesch et al., 2007), полученными в парадигмах, описанных во введении (Klimesch et al., 1997; Pfurtscheller, Aranibar, 1977; Posner, Petersen, 1990). Поскольку степень Д связывают с процессами активации (Pfurtscheller,
Lopes da Silva, 1999; Shaw, 2003), мы можем интерпретировать такие результаты как свидетельство субъективной значимости РС (оцениваемой при вовлечении процессов селективного внимания) при поиске по сравнению с незначимыми нРС, на которых испытуемые совершали фиксации без поиска.

Примерно одинаковая по амплитуде Д во всех отведениях в контроле (рисунки 4, 6), по-видимому, отражает два процесса: сенсорную активацию (непроизвольное внимание 
Рисунок 6

Усредненная по всем испытуемым интегральная величина Д при поиске (темные столбики) и в контроле (светлые столбики) по всем отведениям

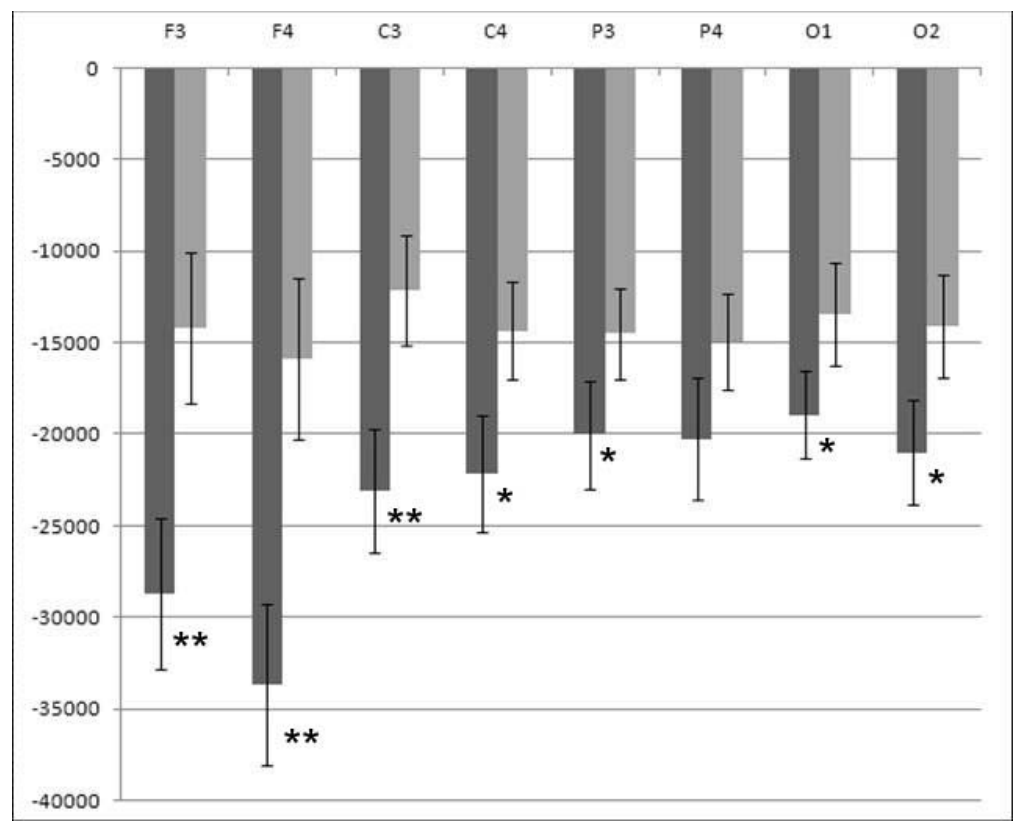

Примечание. Интегральную величину рассчитывали как модуль суммы всех бинов отрицательной амплитуды (в \% ${ }^{2}$ ), приведенных на графиках на рисунке 4. Разброс данных представлен ошибкой среднего. Достоверность различий (по парному критерию Стьюдента): * $-p<0.05{ }^{* *}-p<0.001$. Различие для отведения Р4 оказалось близким к достоверному $(p<0.107)$.

«снизу-вверх» при действии любого стимула) и подавление движений глаз (в соответствии с инструкцией «совершать фиксацию на стимулах»).

Большая выраженность Д при поиске, по-видимому, связана с дополнительными процессами, связанными со значимостью стимула (вовлечение произвольного внимания «сверху-вниз»). Причем такая дополнительная активация составляет около $50 \%$ от активации в контроле. При этом еще большая (в 2 раза по сравнению с контролем) дополнительная Д в лобных областях предположительно отражает значимость выполненной двигательной программы (внимание «сверху-вниз»).

Для разделения перечисленных процессов необходимы дальнейшие исследования. Например, для выделения компонентов активации, связанных с выполнением двигательной программы, необходимо провести исследования по парадигме oddball. Кроме того, чтобы отделить когнитивные процессы (выбор РС) от двигательных программ, необходимо провести специальный эксперимент, в котором испытуемые будут выполнять только двигательные программы без выбора каких-либо стимулов. 


\section{Литература}

Кулаччев А.П. Компьютерная электрофизиология. М.: Изд-во Моск. ун-та, 2002.

Delorme A., Makeig S. EEGLAB: an open source toolbox for analysis of singletrial EEG dynamics including independent component analysis // J. Neurosci. Methods. 2004. 134. 9-21.

Duncan J., Humphreys G. Visual search and stimulus similarity // Psychological Review. 1989. 96. 433-458.

Findlay J.M. Saccade target selection during visual search // Vision Res. 1997. 37. 617-631.

Gomarus H.K., Althaus M., Wijers A.A., Minderaa R.B. The effects of memory load and stimulus relevance on the EEG during a visual selective memory search task: an ERP and ERD/ERS study // Clin. Neurophysiol. 2006. 117. 871-884.

Hwang A.D., Higgins E.C., Pomplun M. A model of top-down attentional control during visual search in complex scenes // J. of Vision. 2009. 9. 5. 1-18.

Klimesch W., Doppelmayr M., Pachinger $T$., Russegger H. Event-related desynchronization in the alpha band and the processing of semantic information // Cogn. Brain Res. 1997. 6. 83-94.

Klimesch W., Sauseng P., Hanslmayr S. EEG alpha oscillations: the inhibition-timing hypothesis // Brain Res. Rev. 2007. 53. 63-88.

Lindsley D.B. Attention, consciousness, sleep and wakefulness // J. Field, H.W. Magoun, V.E. Hall (eds.). Handbook of Physio- logy. Neurophysiology III. Amer. Physiol. Soc. Washington, DC: 1960. P. 1553-1593.

Motter B. C., Holsapple J. Saccades and covert shifts of attention during active visual search: Spatial distributions, memory, and items per fixation // Vision Res. 2007. 47. 1261-1281.

Pfurtscheller G., Aranibar A. Event-related cortical synchronization detected by power measurements of scalp EEG // Electroencephalogr. Clin. Neurophysiol. 1977. 42. 817-826.

Pfurtscheller G., Klimesch W. Functional topography during a visuoverbal judgement task studied with event-related desynchronization mapping // J. Clin Neurophysiol. 1992. 9. 120-131.

Pfurtscheller G., Lopes da Silva F.H. Event-related EEG/MEG synchronization and desynchronization: basic principles // Clin. Neurophysiol. 1999. 110. 1842-1857.

Posner M.I. Orienting of attention // Quarterly J. of Exp. Psychol. 1980. 32. 3-25.

Posner M.I., Petersen S.E. The attention system of the human brain // Ann. Rev. Neurosci. 1990. 13. 25-42.

Shaw J.S. The brain's alpha rhythms and the mind. B.V. Elsevier Science, 2003.

Walter G.W. Can attention be defined in physiological terms? // Evans C.R., Malholland T.B. (eds.). Attention in Neurophysiology. London: Butterworth, 1969. P. 27-39.

Wolfe J.M. Guided search 20: A revised model of visual search // Psychonomic Bulletin \& Review. 1994. 1. 202-238. 